\title{
Identificando tipografias impressas na Revista de Pernambuco
}

\author{
Identifying typefaces printed in Revista de Pernambuco \\ Loudovico Soares, Lucas Arcanjo, Bruna Oliveira, Vanessa Macedo e Isabella Ribeiro \\ Aragão
}

tipografia, composição de tipos, história da tipografia brasileira, Revista de Pernambuco

\begin{abstract}
O presente artigo apresenta uma pesquisa analítica sobre as tipografias impressas em algumas páginas das edições da Revista de Pernambuco de 1926. A pesquisa almejou identificar os tipos, relacionando-os com o contexto histórico e buscando a origem dos desenhos em catálogos de fundições nacionais e internacionais atuantes no início do século XX. Utilizando métodos de análise comparativos entre as tipografias da revista e catálogos dessas fundições foi possível identificar alguns desenhos da Funtimod, a maior fundição nacional do século XX, e apontar a necessidade de estudos sobre os tipos que circulavam no Brasil.
\end{abstract}

typography, type setting, Brazilian typographic history, Revista de Pernambuco

This paper presents an analytical research about the typefaces printed in some pages of Revista de Pernambuco's editions from 1926. The research aimed to identify the typefaces, relating them to their historic context and tracking their origins in specimens of national and international foundries operating in the early twentieth century. By using comparative analytical methods between the magazine's typefaces and the specimens' ones it was possible to identify some designs from Funtimod, the largest Brazilian type foundry from the twentieth century, and to point out the need for studies about types commercialized in Brazil.

\section{Introdução}

Tendo como plano de fundo um cenário onde melhorias substanciais na tecnologia tipográfica foram estabelecidas e o potencial de variedade de desenhos de tipos era explorado pelas fundições (Meggs, Purvis, 2009), esta pesquisa ${ }^{1}$ teve como objetivo principal analisar e identificar alguns tipos impressos na Revista de Pernambuco em 1926. O principal método utilizado no reconhecimento dos desenhos das letras foi a comparação de fontes produzidas no período com as compostas nos textos do artefato. Dessa forma, conseguimos localizar a fundição responsável pela fabricação de alguns tipos, assim como conhecer alguns desenhos disponíveis para composição de texto no começo do século XX no Brasil.

A Revista de Pernambuco, cujas edições encontram-se na Biblioteca Central Blanche Knopf da Fundação Joaquim Nabuco, foi uma revista de publicação mensal, publicada entre 1924 e 1926, que apresentava matérias sobre as comemorações mais importantes do estado. Sua primeira edição surgiu em 2 de julho de 1924, idealizada pelo corpo redacional do Diário do Estado e editada pela Repartição de Publicações Oficiais (Macedo, 2009).

\section{Metodologia de identificação dos tipos}

A metodologia de identificação dos tipos da Revista de Pernambuco foi dividida em duas etapas, a primeira etapa consistiu na seleção do objeto de estudo e coleta de dados; e a segunda etapa esteve relacionada com a análise dos tipos selecionados.

\footnotetext{
${ }^{1}$ A pesquisa foi realizada durante a disciplina de História da Tipografia do Bacharelado em Design da UFPE, ministrada pela professora Isabella R. Aragão, durante o primeiro semestre de 2017.

Anais do ${ }^{\circ} \mathrm{CIDI}$ e 8 CONGIC

Guilherme Santa Rosa; Cristina Portugal (orgs.)

Sociedade Brasileira de Design da Informação - SBDI

Natal | Brasil | 2017

ISBN 978-85-212-1305-5
} 


\section{Coleta de dados}

O volume compilado com todas as edições da Revista de Pernambuco do ano de 1926, da Biblioteca da Fundação Joaquim Nabuco, foi observado no intuito de selecionar algumas páginas com uma boa variedade de tipografias diferentes. Para que a pesquisa não ficasse muito extensa, foram escolhidos alguns anúncios de duas páginas das revistas e uma página com a matéria "Echos da Excursão a Pesqueira" para serem estudados (figuras 1 e 2). Assim sendo, a pesquisa abrangeria tanto tipos de texto quanto tipos displays.

Figura 1: Página escolhida como objeto de estudo.

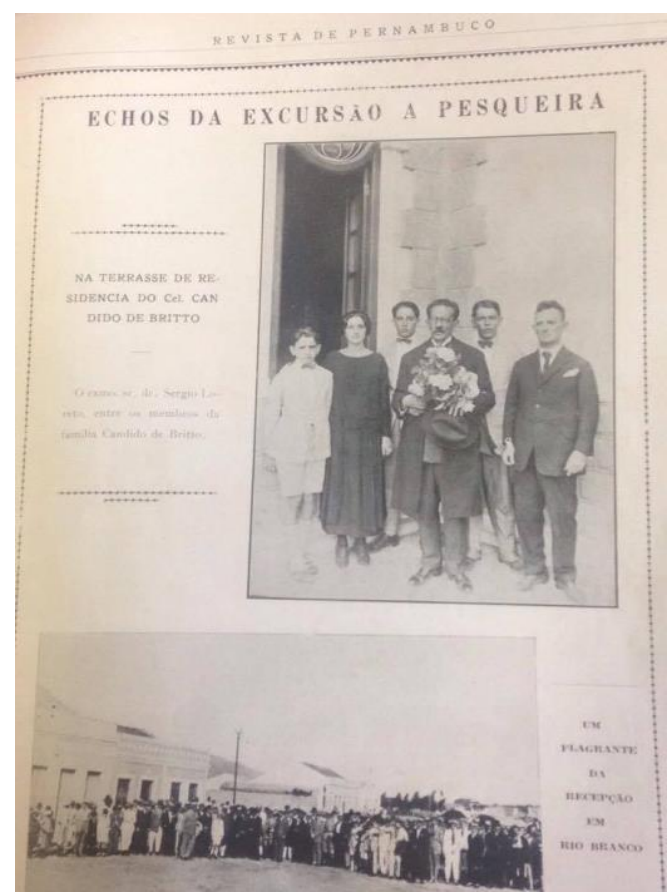

Figura 2: Anúncios analisados na pesquisa.

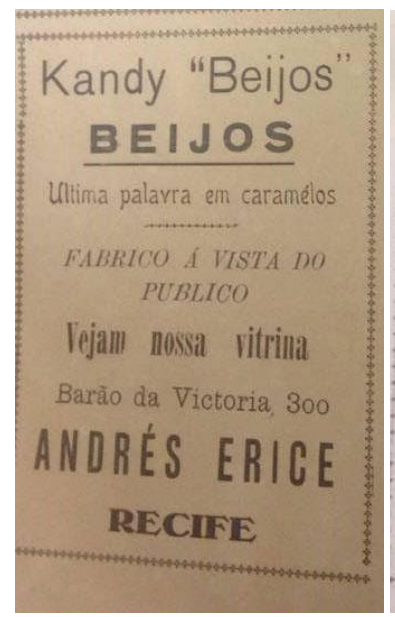

\section{Fabrica Favorita}

BOMBONS E CARAMELOS FRAGOSO \&s C.ia

Praça do Mercado, 123, 127 e 131

Endereço: "FIVORITI" Telephone: 25.92

Recife - Pernambuco

A fase de coleta de dados foi finalizada com a reprodução fotográfica dos impressos, para em seguida iniciar a análise dos tipos, que foi dividida em separação e identificação dos tipos.

\section{Análise dos dados}

Primeiramente, as páginas foram observadas e os tipos distintos entre si foram separados em grupos de acordo com suas características formais (Figura 3), utilizando como base dois dos oito atributos definidos por Dixon (2008) em seu sistema de descrição tipográfica: terminação e modelagem. Outros atributos - forma, peso, proporção e caractere-chave - ajudaram no 
processo de identificação em etapas posteriores de comparação entre os tipos analisados e os tipos presentes em catálogos de tipos.

Portanto, o grupo dos 15 tipos diferentes impressos na revista foram subdivididos em tipos sem serifa e tipos com serifa, assim como em hastes com espessuras diferentes e hastes com espessuras iguais. Dessa forma, acreditamos que a atividade de busca por tipografias similares seria realizada com mais facilidade.

Figura 3: Separação dos tipos por características comuns, com enumeração.
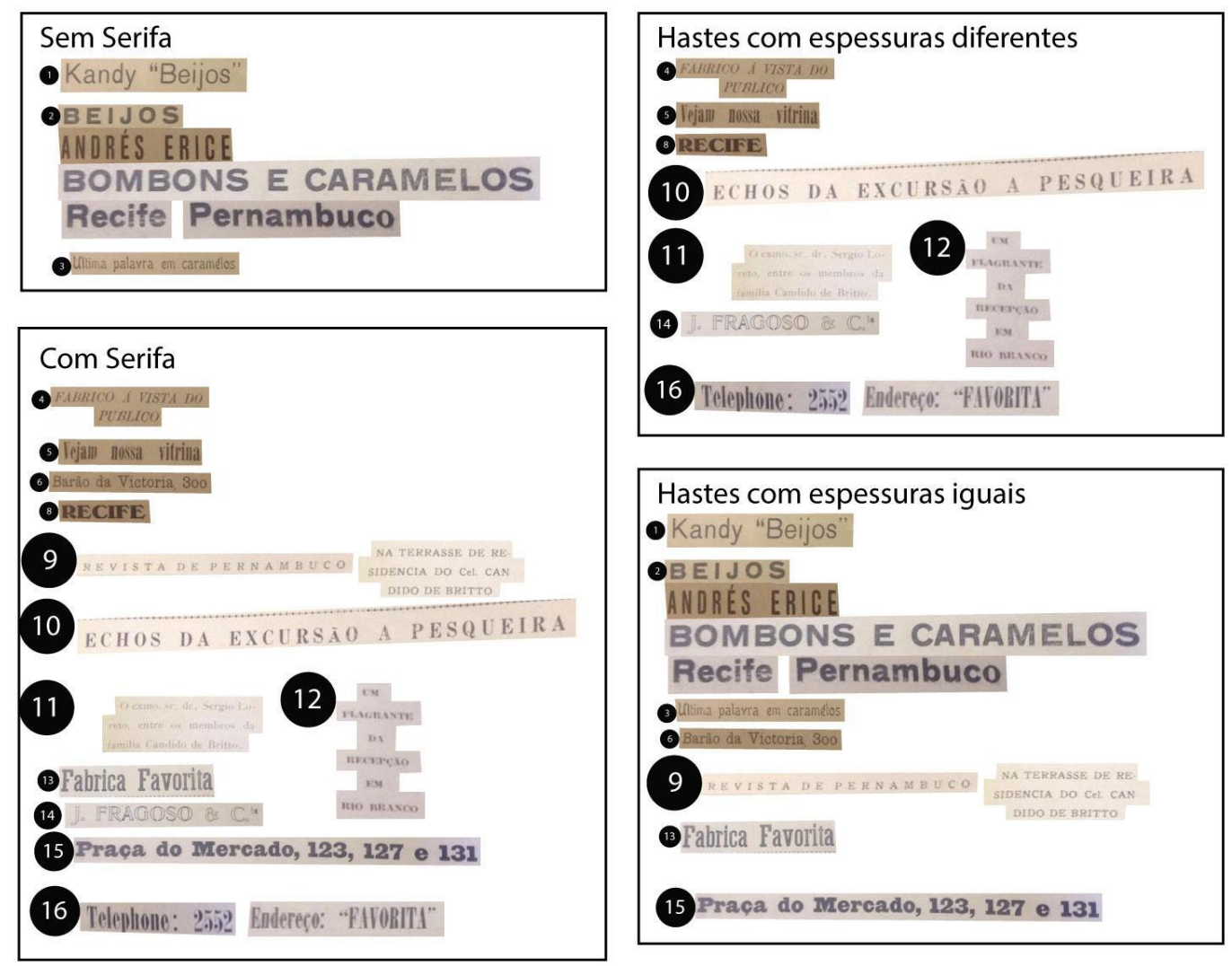

Para a identificação das tipografias já coletadas nos impressos e separadas por características comuns foram utilizados dois métodos comparativos de análise, definidos pelos pesquisadores: método de comparação por fisionomia e método de comparação por sobreposição.

O primeiro método, de comparação por fisionomia, consistiu em uma busca mais abrangente por tipografias com os mesmos atributos. Ou seja, procuramos identificar os tipos da revista a partir da observação comparativa de suas características com as tipografias de catálogos de tipos de metal de fundições brasileiras e estrangeiras disponíveis.

A maioria dos catálogos das fundições pesquisadas pertencem a biblioteca particular de Aragão (2016), por exemplo, Funtimod, Manig, Monotype, Linotype, entre outros. Ademais, outras referências foram encontradas digitalizadas e disponíveis online, como a catalogação de tipos alemães do site do Klingspor Museum².

Quando as fontes estudadas não eram encontradas nos catálogos, utilizávamos sistemas de identificação de fontes online, como o "What the font?" e o "Identifont". Nesses mecanismos, os métodos variam entre fornecimento de informações sobre as características anatômicas do tipo ou envio de imagens digitalizadas dos mesmos.

No que concerne ao método de comparação por sobreposição, comparamos digitalmente os desenhos digitalizados dos catálogos de tipos e os exemplares da revista. Este método somente era executado depois de resultados positivos do método de comparação por

\footnotetext{
${ }^{2}$ http://www.klingspor-museum.de/Intl_Bleisatz_Index.html
} 
fisionomia. No entanto, como não tínhamos as páginas da revista digitalizadas, as conclusões sobre a atividade de identificação somente foram realizadas com a sobreposição manual entre os desenhos das fundições impressos em transparência e as páginas da própria revista, na Biblioteca da Fundação Joaquim Nabuco.

Algumas sobreposições entre os impressos foram realizadas com a ajuda de um microscópio digital. Com uma visão mais aproximada (entre 20 e 200 vezes) foi possível comprovar quais tipos realmente eram iguais e quais apresentavam pequenas diferenças.

\section{Resultado da identificação dos tipos}

Em relação à identificação, entre os 15 desenhos que compuseram os textos das páginas selecionadas da Revista de Pernambuco apenas três tipos foram encontrados nos catálogos da fundição brasileira Funtimod, conforme resultado da Tabela 1. Na tabela apresentamos uma amostra dos tipos, a fonte e fundição identificadas.

\begin{tabular}{|c|c|c|c|}
\hline Fonte & Amostra do objeto de estudo & Fonte identificada & $\begin{array}{l}\text { Fundição } \\
\text { identificada }\end{array}$ \\
\hline 1 & Kandy "Beijos" & $\begin{array}{l}\text { Grotesca Normal Clara } \\
\text { Corpo } 36\end{array}$ & $\begin{array}{l}\text { Funtimod } \\
\text { (1983?) }\end{array}$ \\
\hline 2 & $B E 1.108$ & $\begin{array}{l}\text { Parecida com Grotesca } \\
\text { Larga Meia Preta da } \\
\text { Funtimod (1983?) }\end{array}$ & \\
\hline 3 & Ullima palavra em caramélos & $\begin{array}{l}\text { Parecida com a Reklame, } \\
\text { Moderne (Seemann, 1926, } \\
\text { p. 169) }\end{array}$ & \\
\hline 4 & FABRLCO A TISTA DO & $\begin{array}{l}\text { Antiga Oficial Grifo } \\
\text { Corpo } 12\end{array}$ & $\begin{array}{l}\text { Funtimod } \\
\text { (1983?) }\end{array}$ \\
\hline 5 & 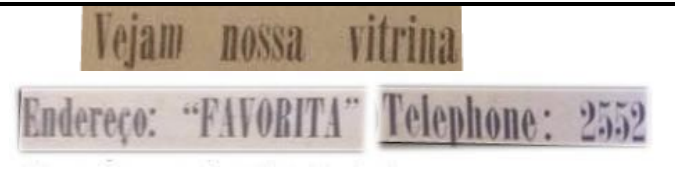 & $\begin{array}{l}\text { Parecida com a Bodoni } \\
\text { Poster compressed }\end{array}$ & \\
\hline 6 & Barão da Victoria 300 & & \\
\hline 7 & ANBRES ERIGE & $\begin{array}{l}\text { Parecida com a Grotesca } \\
\text { Reforma Meia Preta } \\
\text { Estreita da Funtimod } \\
\text { (1983?) }\end{array}$ & \\
\hline 8 & RECIFE & $\begin{array}{l}\text { Bravour meio preto } \\
\text { Corpo } 16\end{array}$ & $\begin{array}{l}\text { Funtimod } \\
\text { (1983?) }\end{array}$ \\
\hline 9 & REVISTA DE PERNAMBUCO & $\begin{array}{l}\text { Parecida com a Excelsior } \\
\text { corpo } 10 \text { da Funtimod } \\
\text { (1983?) }\end{array}$ & \\
\hline 10 & ECHOS DA EXCURSÃO A PESQ & & \\
\hline 11 & 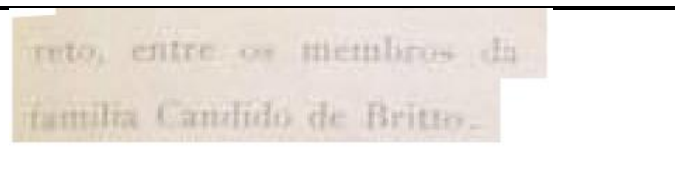 & $\begin{array}{l}\text { Parecida com a Excelsior } \\
\text { corpo } 8 \text { da Funtimod } \\
\text { (1983?) }\end{array}$ & \\
\hline 12 & H. Mettisir. & & \\
\hline
\end{tabular}




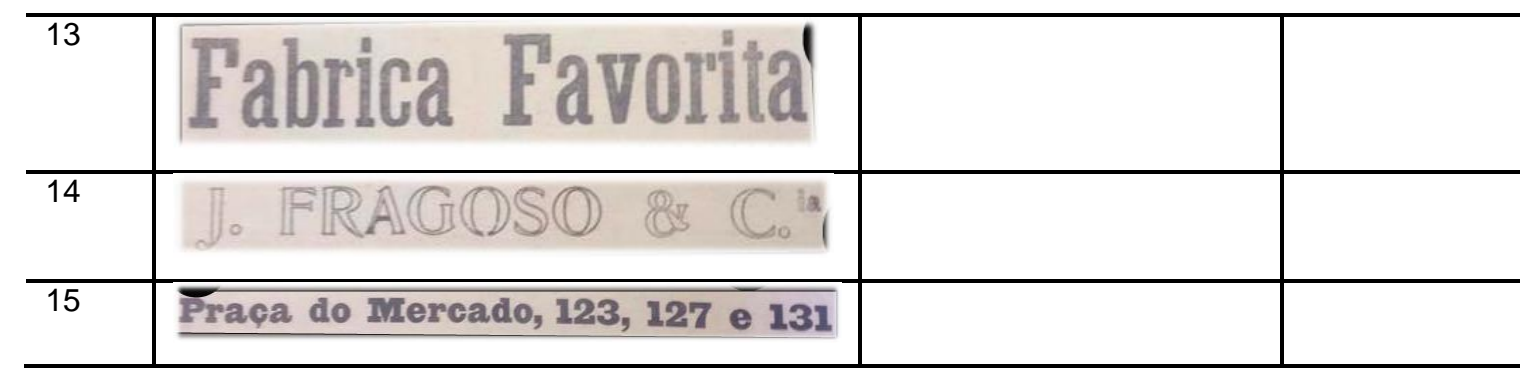

\section{Discussão dos resultados da identificação dos tipos}

A tipografia Grotesca Normal Clara corpo 36, da Funtimod, foi identificada como a fonte 1 da separação por meio da sobreposição manual (transparência e revista), que ainda foi observada com o microscópio (Figura 4). Nas imagens tiradas com o microscópio é possível perceber um perfeito encaixe entre os desenhos da letra "a" da palavra "Kandy".

Figura 4: Sobreposição central da letra "a" à esquerda e sobreposição deslocada à direita

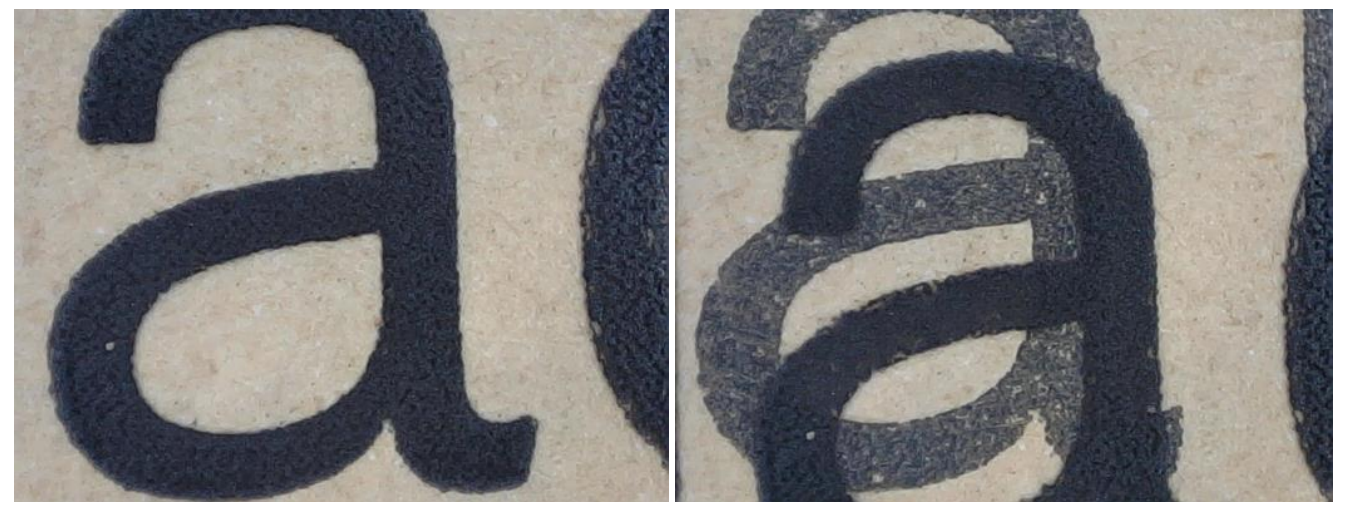

No arranjo da mesma frase destacamos uma pequena peculiaridade na composição da palavra "Beijos": aspas diferentes (Figura 5). Embora não tenha sido possível confirmar se um dos dois tipos de aspas pertence à Grotesca Normal Clara, essa diversidade de desenhos do mesmo caractere na mesma palavra, que indica o uso de composição tipográfica manual, ratificou a identificação do tipo da Funtimod, que produzia apenas tipos móveis.

Figura 5. Aspas diferentes na palavra "Beijos" e caracteres ampliados com microscópio.
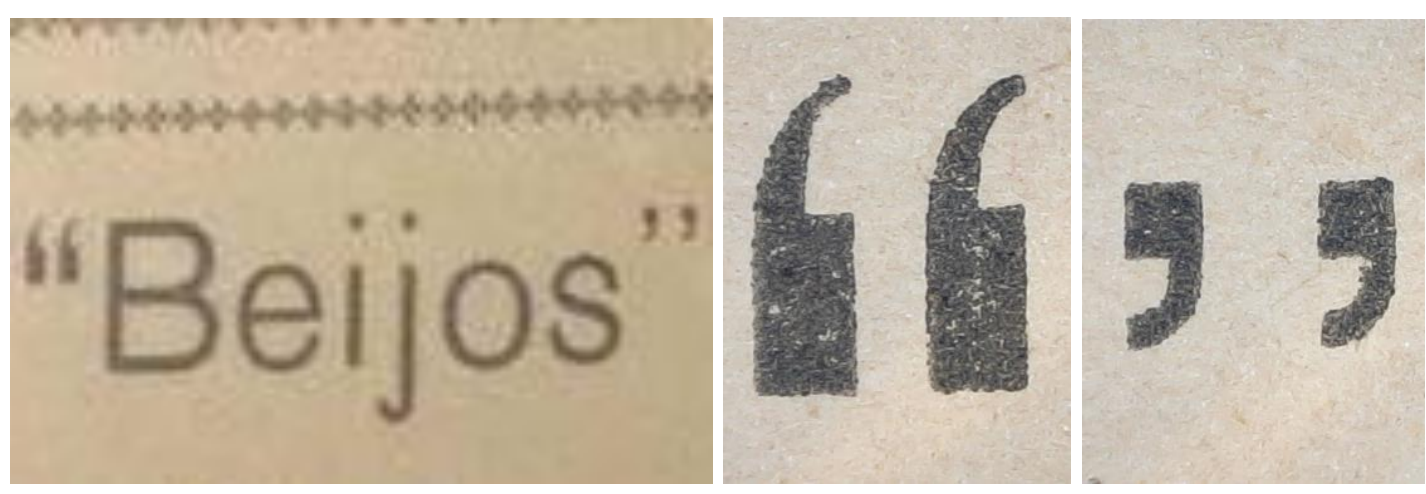

A Antiga Oficial Grifo 12 pts e a Bravour Meio Preto 16 pts, ambas da Funtimod, compuseram, respectivamente, a fonte 4 e a fonte 8 no mesmo anúncio da revista. De modo igual que na fonte 1 , observamos o uso de composição manual na frase "fabrico á vista do 
publico" da fonte 4 do anúncio, os desenhos das letras "B" e "O" são diferentes entre si (figura 6). Estas mudanças de desenhos do mesmo caractere numa frase, além de ajudar a identificar tipos em fundições que produziam tipos móveis, também podem ser relacionadas com práticas da seção de composição da tipografia, por exemplo, escassez na quantidade de tipos, caixa tipográfica misturada e/ou falta de atenção do compositor.

Figura 6. Ampliação com microscópio das letras "B" e "O" compostas na mesma frase do anúncio da Revista de Pernambuco.

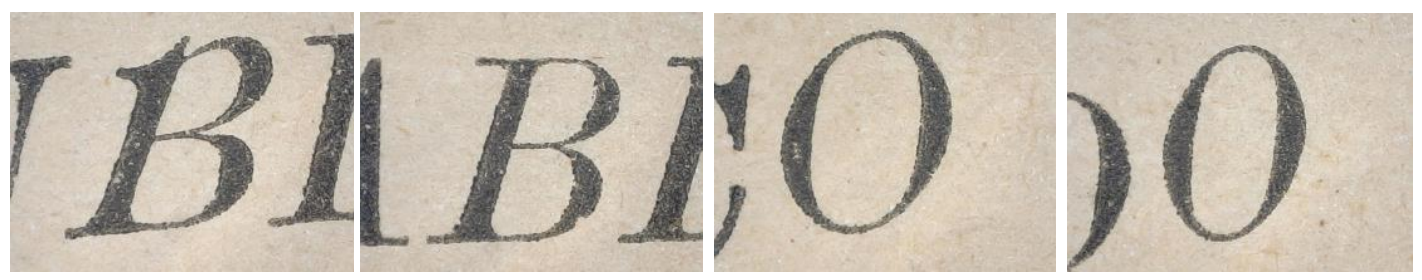

As fontes 9 e 11 foram identificadas como a Excelsior da Funtimod durante o método de comparação por fisionomia. No entanto, resultados negativos foram alcançados após observarmos diferenças nas letras (Figura 7) durante a aplicação do método de sobreposição com o auxílio do microscópio.

Figura 7: Comparações entre a fonte 9 da revista (baixo) e a Excelsior da Funtimod (cima) observadas com microscópio.
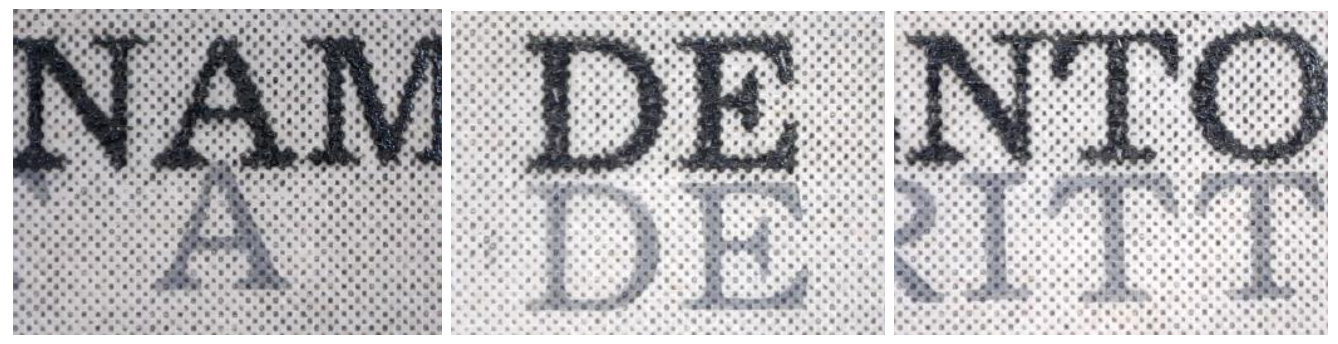

O resultado da identificação dos tipos da Revista de Pernambuco aponta a necessidade de reflexão sobre duas questões. A primeira diz respeito a uma grande parcela de desenhos não identificados e a segunda se relaciona com a fundação da Funtimod.

Algumas tipografias não foram identificadas durante o processo, contudo, foi possível encontrar, em outras fontes que não os catálogos estudados, resultados de desenhos aproximados, como as grotescas das fontes 2 e 7. Esta última tem algumas letras similares à Grotesca Reforma Meia Preta Estreita, da Funtimod, ao passo que tem outras muito diferentes. Outra fonte que resultou em uma identificação muito aproximada foi a fonte 5 , que é parecida, mas não idêntica, à Bodoni Poster Compressed.

Outros desenhos mais ornamentados, como as fonte 3 e 14, provavelmente, estão mais próximos dos tipos populares no século XIX. Segundo a listagem final dos tipos produzidos neste século do livro de Gray (1951), é possível encontrar vários tipos vazados sendo produzidos pelas fundições inglesas.

Os tipos não identificados ou foram fabricados no Brasil por fundições atuantes entre o final do século XIX e início do século XX, ou foram importados de fundições estrangeiras. $A$ escassez de estudos sobre tipografia no Brasil e a dificuldade de acesso a catálogos de tipos dessa época dificultam esses processos de identificação e, em consequência, o entendimento desta parte da história da tipografia nacional.

Segundo Aragão (2016), a Funtimod foi fundada apenas em 1932. Se a Revista de Pernambuco foi impressa em 1926, como seus tipos foram identificados no catálogo da fundição brasileira? Conforme a pesquisadora (Aragão, 2016), havia uma grande comercialização de matrizes de tipos no século 20, então, os mesmos tipos já poderiam estar 
sendo fabricados por outras empresas brasileiras e/ou estes podem ter pertencido às empresas adquiridas pela Funtimod para sua criação.

\section{Considerações Finais}

Apesar da dificuldade em encontrar todos os tipos, algumas tipografias foram identificadas no catálogo da Funtimod (1983?) e outras fontes tiveram resultados aproximados a tipos da empresa e do livro Handbuch der Schriftarten (Seemann, 1926) que cataloga tipos alemães.

Quanto aos atributos formais das fontes utilizadas nas composições dos anúncios, observamos uma grande variedade de desenhos tipográficos, com maior presença de tipos com serifa. Como a revista foi impressa em 1926 com desenhos mais semelhantes aos produzidos no século XIX, podemos apontar uma desatualização do gosto tipográfico. A partir do século $X X$, os tipos sem serifa se tornaram mais populares e as composições utilizavam uma variedade menor de tipografias.

Observar o processo de composição tipográfica manual nas páginas impressas pode tanto ajudar na atividade de identificação dos tipos, na medida que direciona a busca para fundições de tipos móveis, quanto revelar práticas das oficinas tipográficas brasileiras.

O resultado da pesquisa demonstra a necessidade para estudos mais aprofundados na área de fundição de tipos em território nacional no início do século XX visto que poucos tipos foram identificados; além de despertar a curiosidade a respeito dos tipos identificados nos catálogos da Funtimod e das principais fundições que exportavam tipos para o Brasil naquele período.

\section{Referências}

ARAGÃO, I. R. 2016. Tipos móveis de metal da Funtimod: contribuições para a história tipográfica brasileira. 2016. Tese (Doutorado em Design e Arquitetura) - Faculdade de Arquitetura e Urbanismo, University of São Paulo, São Paulo, 2016. Disponível em: $<$ http://www.teses.usp.br/teses/disponiveis/16/16134/tde-01092016-154117>.

DIXON, C. 2008 Describing typeforms: a designer's response. Infodesign, v 5, n 2, SBDI: São Paulo, 2008.

FUNTIMOD S.A. - Máquinas e materiais gráficos. 1983?. São Paulo: Funtimod.

GRAY, N. 1951. XIXth century ornamented types and title pages. Londres: Faber and Faber.

MACEDO, A. C. 2009 Propagandas em Revistas Recifenses das Décadas de 1910 a 1950: Catálogo. [Recife]: Fundação Joaquim Nabuco, Biblioteca Central Blanche Knopf.

MEGGS, P. B.; PURVIS, A. W. 2009 História do Design Gráfico, 1. ed. São Paulo: Cosac Naify. SEEMANN, A. 1926. Handbuch der Schriftarten. Leipzig: Verlag.

\section{Sobre os autores}

Loudovico Soares, estudante do Bacharelado em Design, UFPE, Brasil <vicosoares.designer@gmail.com>

Lucas Arcanjo Soares, estudante do Bacharelado em Design, UFPE, Brasil <arc_lucas@hotmail.com>

Bruna Oliveira Soares, estudante do Bacharelado em Design, UFPE, Brasil $<$ brunabo.bbo@gmail.com>

Vanessa Macedo Soares, estudante do Bacharelado em Design, UFPE, Brasil $<$ vanessa.macedo.15@gmail.com>

Isabella Ribeiro Aragão, Doutora, UFPE, Brasil <isabella.aragao@gmail.com> 